\title{
Reconceptualizing the Experience of Surrogate Decision Making: Reports vs Genuine Decisions
}

\author{
Ursula K. Braun, MD, MPH ${ }^{1,2}$ \\ Aanand D. Naik, $M D^{1,2}$ \\ Laurence B. McCullough, $\mathrm{PbD}^{3}$ \\ 'Houston Center for Quality of Care and \\ Utilization Studies, Michael E. DeBakey VA \\ Medical Center, Houston, Texas
}

${ }^{2}$ Sections of Geriatrics and Health Services Research, Department of Medicine, Baylor College of Medicine, Houston, Texas

${ }^{3}$ Center for Medical Ethics and Health Policy, Baylor College of Medicine, Houston, Texas
Conflicts of interest: none reported

\section{CORRESPONDING AUTHOR}

Laurence B. McCullough, PhD

Center for Medical Ethics and Health Policy Baylor College of Medicine

One Baylor Plaza

Houston, TX 77030

mccullou@bcm.edu

\begin{abstract}
BACKGROUND We propose a reconceptualization of surrogate decision making when patients lack an advance directive stating their preferences about lifesustaining treatment. This reconceptualization replaces the current 2-standard model of substituted judgment (based on the patient's prior preferences and values) and best interests (an assessment of how best to protect and promote the patient's health-related and other interests).
\end{abstract}

METHODS We undertook a conceptual analysis based on the ethics of informed consent, a qualitative study of how surrogates of seriously ill patients experience the surrogate's role, and descriptions of decision making.

RESULTS When the surrogate can meet the substituted judgment standard, the experience of the surrogate should be understood as providing a report, not making a decision. Surrogate decisions based on the best interest standard are experienced as genuine decisions, and the label "surrogate decision making" should be reserved to characterize only these experiences.

CONCLUSIONS Physicians should identify clinically reasonable options and elicit the surrogate's sense of decision-making burden. Some surrogates will be able to make reports, and the physician should make a clear recommendation that implements the patient's reported preference. Some surrogates will confront genuine decisions, which should be managed by negotiating treatment goals. Requests by the surrogate that everything be done may represent a psychosocially burdensome decision, and support should be provided to help the surrogate work through the decision-making process.

Ann Fam Med 2009;7:249-253. DOI: 10.1370/afm.963.

\section{INTRODUCTION}

E thical challenges can arise regarding the use of life-sustaining treatment for seriously ill patients when the patient can no longer participate in decision making about the initiation, continuation, or discontinuation of such interventions, and when the patient has not left an advance directive stating his or her preferences regarding life-sustaining treatment. Current practice in these cases is that family members or others legally sanctioned to do so will act as surrogates and make decisions for the patient. ${ }^{1}$ Surrogate decision making has been legally recognized at least since In re Quinlan in 1976, when the New Jersey Supreme Court ruled that an adult patient's right to make decisions becomes meaningless if that right cannot be exercised by surrogates at the appropriate time. ${ }^{2}$ Since the Quinlan decision, the first end-of-life case to be adjudicated, advance directives legislation has codified surrogate decision making. ${ }^{3}$

Two standards should guide surrogate decision making. ${ }^{1}$ The substituted judgment standard calls for surrogate decision making to be based on the incapacitated patient's known preferences and values and the surrogate's level 
of confidence that these preferences and values apply to the patient's condition and its prognosis. ${ }^{1}$ If relevant preferences and values cannot be identified with confidence, decisions should be guided by an assessment of how best to protect and promote the patient's interests, known as the best interest standard of surrogate decision making. ${ }^{1}$ In the application of this standard, there are no presumptions that the patient would prefer continuing life-sustaining treatment or that such treatment is always required to protect and promote the patient's interests

A recent study reports qualitative data from a sample of African-American, white, and Hispanic participants who served as surrogate decision makers for seriously ill patients. ${ }^{4}$ All 3 groups emphasized the heavy burden of surrogate decision making, an important aspect that previously has been poorly characterized. In this study, the burden was attributed to uncertainty about the prognosis of continued or increased life-sustaining treatment, uncertainty about the patient's preferences, or both. Furthermore, this burden was inversely proportional to the surrogate's confidence in his or her judgment about the patient's preferences. When judgments met what Buchanan and Brock call "high evidentiary standards,"1 eg, when the patient had spoken directly to the issue of life-sustain- ing treatment and expressed a very clear preference for or against it, and the surrogate was very sure about the patient's wishes, the surrogate's role was no longer perceived to be that of a "decision maker." Instead, the role was perceived to be that of a "reporter" of the patient's preference, or even an enforcer, ie, seeing to it that these preferences were implemented.

These findings suggest that the current model of surrogate decision making should be reconceptualized to better address the complex roles and responsibilities of the decision-making experience. The purpose of our article is to describe and argue for a reconceptualization that more adequately characterizes this experience than does the current model, which assumes that surrogate decision makers uniformly experience their role as making a decision from among feasible options (Figure 1). We believe that putting this reconceptualization into clinical practice will provide practical direction to clinicians engaged in the difficult task of talking with patients' families about setting expectations for intervention at the end of life.

\section{ETHICAL AND CONCEPTUAL ANALYSIS}

A standard tool of medical ethicists is conceptual analysis, undertaken to clarify key concepts as the basis for

\section{Figure 1. Reconceptualizing the experience of surrogate decision making.}

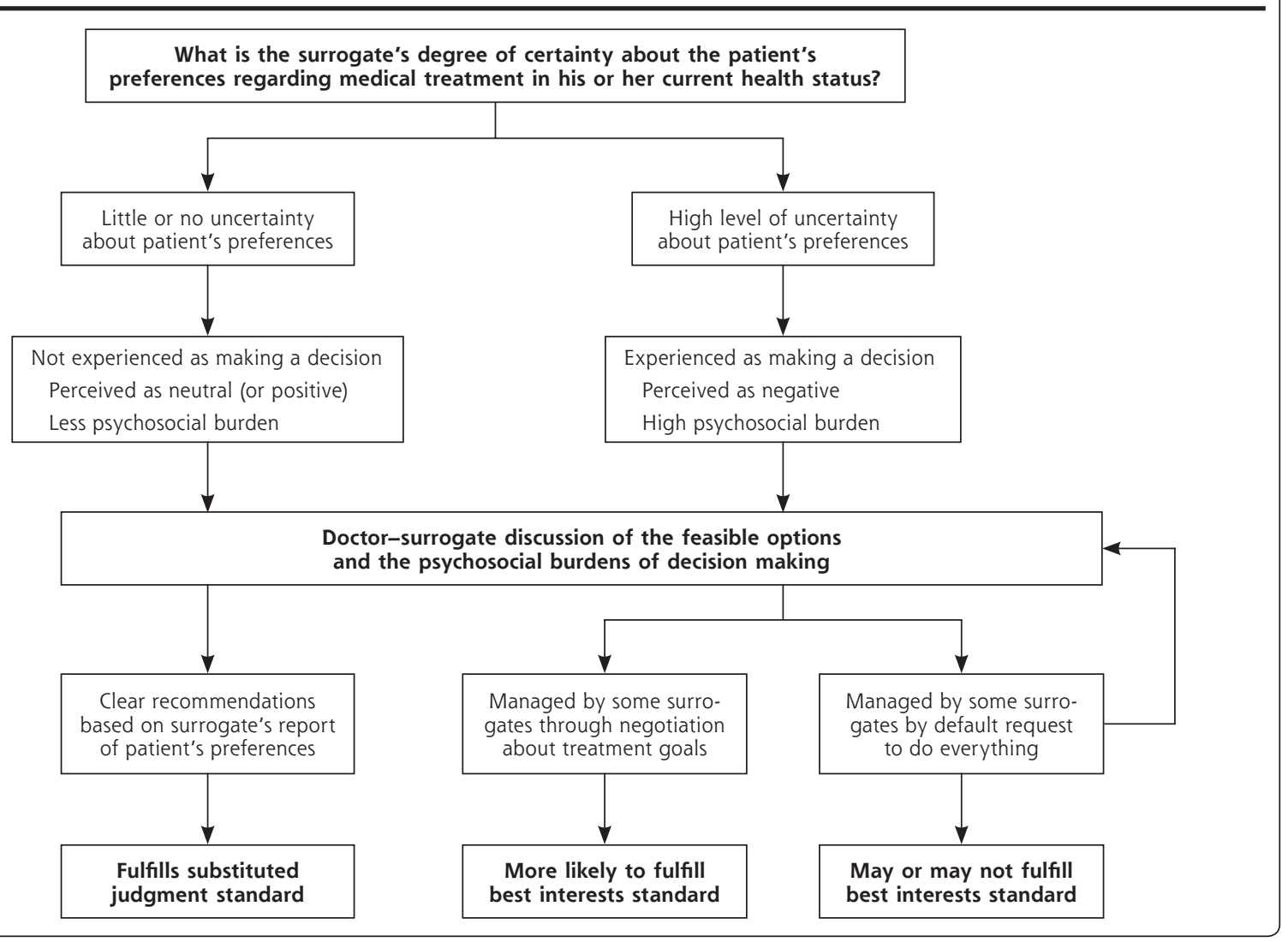


their application to guide clinical practice. We provide a brief account of the physician's disclosure obligation in the informed consent process, which has been well described in the medical ethics literature, and of the ensuing decision-making process, which has not been as well described in the medical ethics literature.

\section{Physician's Disclosure Obligation in the Informed Consent Process}

The physician has the ethical and legal obligation to start the informed consent process by presenting to the decision maker the medically reasonable alternatives for the management of the patient's condition. ${ }^{5}$ The physician should identify technically possible, medically reasonable, and available interventions and evaluate each, making an evidence-based judgment about which alternatives are expected to result in a net balance of clinical good over clinical harm. These alternatives and should then be presented to the decision maker, along with an account of their benefits and risks.

\section{Nature of Decision Making}

The medical ethics literature on surrogate decision making does not describe explicitly the ensuing decision-making process. Buchanan and Brock are representative in their implicit understanding of decision making, which appears to have 3 components. ${ }^{1}$ First, decisions about medical care focus on the clinical management of a patient's condition. A sufficient grasp by the patient or surrogate of the relevant diagnostic information presented by the physician is the first step in the decision-making process. ${ }^{1(\mathrm{p} 23)}$ Second, the physician makes a recommendation. Third, the patient or surrogate assesses the alternatives, including the recommended alternative, on the basis of the patient's values. ${ }^{1(\mathrm{p} 25)}$ A decision occurs when a selection is made from the medically reasonable alternatives.

Faden and Beauchamp, in their pioneering study of informed consent, understand the exercise of the patient's autonomy to occur in 3 components: intentionality (acting according to a plan); understanding; and noncontrol, or voluntariness (freedom from substantially controlling or coercive influences). ${ }^{5}$ Voluntariness is conceptually important for autonomous decision making because substantial control or coercion can occur when a patient is denied information about all medically reasonable alternatives and is presented, in effect, with only one choice. Control or coercion can also occur when the patient is directed to select one alternative, eg, under the pressure of paternalism. Voluntariness can also be impaired by internal factors, such as guilt, anger, or fear.

Byrne identifies a 4 -step account of decision making: ${ }^{6}$ The first step involves setting a goal, the second step compiles the options for producing that goal. The third step involves ranking the options in the order of preference, and the last step involves selecting the highest ranked alternative. In everyday situations, of course, decision making is generally more dynamic and recursive than steplike. ${ }^{6(\mathrm{p} 209)}$

Byrne's account is akin to that of goal-setting theory. ${ }^{7,8}$ In health care decision making, the clinical goal is effective management of the patient's condition. The options for producing this goal are the medically reasonable alternatives presented by the physician. The patient then ranks these alternatives and selects the highest ranked alternative. Levinson et $\mathrm{al}^{9}$ have shown that patients expect physicians to identify and present medically reasonable alternatives for consideration. Most patients are willing to engage in a discussion of the medically reasonable alternatives and their preferences; however, a great portion do not want to assume the authority for making a final decision.

These descriptions can be combined to create an explicit account of decision making. Decisions must be made when the patient or surrogate has more than 1 course of treatment from which to choose. The patient or surrogate evaluates the alternative treatment options on the basis of the patient's values, and then the patient or surrogate voluntarily elects the preferred course of treatment.

\section{SURROGATE REPORTS}

Sometimes surrogates are confident that they have met high evidentiary standards for substituted judgment. Findings strongly suggest, however, that when surrogates are confident in the knowledge of the patient's preferences and values, they do not experience their role as one of making a decision, ie, as having several different options among which they must now choose. ${ }^{4}$ Instead, they can eliminate all alternatives other than the one clearly preferred by the patient (Table 1, Figure 1). Even though there may be medically reasonable alternatives, such as continuing current levels of lifesustaining treatment, escalating treatment, or choosing palliative treatment, surrogates experience certainty in authorizing only the patient's expressed, preferred clinical management. Failure to provide such authorization is experienced as profound disrespect of the patient and is therefore not considered to be ethically permissible. Surrogates therefore understand they do not to have choices, they do not make a decision.

No decision needs to be made, because the options, as well as the need to select among them, have been eliminated by the well-established previous decisions of the patient. Because multiple options do not need to be separately and comparatively evaluated, reporting the 


\begin{tabular}{|c|c|}
\hline $\begin{array}{l}\text { Surrogate } \\
\text { Position }\end{array}$ & Example \\
\hline Surrogate report & $\begin{array}{l}\text { Mr H had amyotrophic lateral sclerosis diagnosed } 7 \text { years ago. Over the } \\
\text { years he has declared multiple times that he does not want to be kept } \\
\text { alive on a ventilator. He had considerable decline in the last year and } \\
\text { continued to refuse ventilator support. When he goes into respira- } \\
\text { tory distress, he is asked again about his wishes and confirms. When } \\
\text { he becomes severely hypoxic and delirious despite oxygen by mask, } \\
\text { the physician turns to his wife as his surrogate. His wife honors her } \\
\text { husband's wishes saying: "He told me many times that he never wants } \\
\text { to be on a ventilator, and I am carrying out his wishes." }\end{array}$ \\
\hline Surrogate decision & $\begin{array}{l}\text { Mrs } F \text { has severe chronic obstructive pulmonary disease and has recently } \\
\text { been given a diagnosis of stage- } 4 \text { lung cancer that has metastasized to } \\
\text { the liver. She has no advance directives, and her husband never had } \\
\text { conversations with her regarding medical care. When she is admitted } \\
\text { with obstructive pneumonia, she is intubated and requires ventilatory } \\
\text { support. After } 10 \text { days she has not improved. She has suffered a non- } \\
Q \text { wave myocardial infarction and a small cerebrovascular infarct, and } \\
\text { she has developed acute renal failure during the hospital course. Her } \\
\text { husband is asked to decide whether to do a tracheostomy or to treat } \\
\text { her with comfort measures and stop ventilatory support. He asks that } \\
\text { everything be done while he comes to terms with his wife's death. }\end{array}$ \\
\hline
\end{tabular}

sively, to how surrogates experience their role (Figure 1). Reporting a patient's preference should be distinguished from making decisions.

When the substituted judgment standard is met with confidence, clinicians should assume that surrogates understand their role as reporting patients' preferences and should make clear that these preferences will be respected. Physicians should therefore not expect surrogates to make a selection among medically reasonable alternatives. Reports should be understood to be less burdensome than genuine surrogate decision making, and patient's preference reduces burden of responsibility that is associated with genuine surrogate decision making.

Surrogate decision making that satisfies the substituted judgment standard is a conceptually misleading misnomer. Failure to recognize the proposed reconceptualization of the experience of surrogate decision making has important clinical implications.

\section{GENUINE SURROGATE DECISIONS}

Surrogates who cannot with confidence identify the patient's values and preferences for clinical management understand that there is a decision to be made, because the medically reasonable alternatives of continuing, escalating, and discontinuing life-sustaining treatment are now genuine options (Table 1, Figure 1). Data suggest that the burden experienced by surrogates under the best interests standard is directly proportional to their degree of uncertainty regarding the patient's preferences, the consequences of making discordant decisions, and the outcomes of continued or escalated lifesustaining treatment. This uncertainty greatly reduces the surrogate's ability to apply the patient's preferences and values to the treatment options. ${ }^{4}$ The greater the level of uncertainty experienced by the surrogate, the more burdensome the decision that must be made. Surrogates may manage this crisis by electing what is usually the default position in medical practice for managing clinical uncertainty, continued intervention, sometimes expressed as "doing everything."

\section{DISCUSSION}

In clinical practice the concept of surrogate decision making should be applied selectively, not comprehen- they meet the substituted judgment standard of surrogate decision making in medical ethics and law.

Genuine surrogate decision making happens only when the best interests standard must be implemented, ie, when high evidentiary standards cannot be met to the surrogate's satisfaction. Decisions made on the basis of the patient's best interests are indeed experienced as decisions, and they may or may not meet the best interests standard for surrogate decision making.

Much as patients who do not want to assume authority for a final decision, ${ }^{9}$ some surrogates may not welcome the role of genuine surrogate decision maker because of its psychosocial and moral burdens. ${ }^{4}$ In such cases, physicians should not be surprised when surrogates authorize continued life-sustaining treatment or ask that "everything be done." Such requests may not meet the threshold for informed decision making but rather may better be considered coping strategies for alleviating the unwelcome burdens of decision making. This aspect of the experience of surrogate decision making was not identified either in a recent description of the physician-surrogate relationship ${ }^{10}$ or in recent critiques of surrogate decision making. ${ }^{11,12}$ The understandably strong desire to reduce or escape the unwelcome burdens of surrogate decision making may thus result in an element of substantial psychological control.

A request to "do everything" should be addressed in 2 steps. First, the surrogate may be able to make a report but be reluctant to assume the burden of authorizing what the patient would want or not want. To reduce this burden, the physician should point out that the decision has already been made-by the patient. The task at hand is to respect and implement the patient's decision. The surrogate's role is to report the preferences and values of the patient to the physician, 
who assumes responsibility for the final decision based on the patient's preferences and the surrogate's assent.

Second, some surrogates indeed make decisions but struggle with the burden of genuine surrogate decision making. Such a burden is likely to be experienced when the patient is seriously ill and has an uncertain clinical prognosis even with continued treatment, and the surrogate is not confidently able to identify the patient's relevant values and preferences. ${ }^{4}$ In addition, the surrogate may be uncomfortable with having to make such a final decision, especially to discontinue life-sustaining treatment.

Responding initially to a request to "do everything" as an irrational decision unnecessarily increases the risk of preventable ethical conflict between the surrogate and the care team, and such a request could lead to an adversarial relationship between physician and surrogate. Requests to "do everything" should therefore initially be understood as a request for help with managing the decision-making burden (Figure 1).

One antidote to the heavy burden of surrogate decision making is to help surrogates appreciate that in some cases the best interests standard supports not initiating or discontinuing of life-sustaining treatment. Recommending limits on life-sustaining treatment opens this option for the surrogate's serious consideration. This approach may also help identify families whose decisions may be based on religious, cultural, or personal values that admit little or no flexibility, or families that are impervious to clinical facts, the ethical challenges of which are beyond the scope of this article.

The discourse about surrogate decision making and its implicit understanding of surrogates as always being asked to make decisions, just as patients are asked to do, entered medical ethics at a time when conceptual methods predominated in the field. Medical ethics methodology has rapidly expanded to include empirical ethics research. It is now time to articulate how conceptual and empirical methods should inform medical ethics, with neither method assumed to be autonomous. This article contributes a synergistic approach to medical ethics by providing an example of how empirical research on the experience of surrogate decision making informs the need to reconceptualize this major clinical practice, a practice that is supported in ethics, health law, and organizational policy. We propose that the current model be replaced by an empirically derived, conceptually rigorous, and clinically applicable model of the experience of surrogate decision making: making reports, with its lighter psychosocial burdens $;$ and genuine surrogate decision making, with its heavy psychosocial burdens.

On the basis of this new model, physicians should focus not on decision making but on identifying for the surrogate the clinically reasonable options and eliciting the surrogate's sense of the burden of decision making. Some surrogates will be able to make reports, and a clear recommendation should then be made that implements the patient's reported preference. Some surrogates will confront genuine decisions, which should be managed by negotiating treatment goals, addressing requests that everything be done as symptomatic of a psychosocially burdensome surrogate decision, and providing support for the surrogate in working through that burden.

To read or post commentaries in response to this article, see it online at http://www.annfammed.org/cgi/content/full/7/3/249.

Submitted May 13, 2008; submitted, revised, August 26, 2008; accepted September 22, 2008.

Key words: Ethics; surrogate decision making; surrogate reports; surrogate decisions; negotiating

Funding support: This work was supported by the Houston VA Health Services Research and Development Center of Excellence (HFP90-020), Michael E. DeBakey Veterans Affairs Medical Center. Drs Braun and McCullough were supported by VA-IIR-02-224: A Culturally Sensitive Values-Guided Aid for End-of-life Decision Making. Dr Brau was also supported by VA-HSRED-RCDA-02-029: Improving Decision Making for End-of-Life Care. Dr Naik was supported by grants from NIH (K23AG027144) and the Greenwall Foundation.

\section{References}

1. Buchanan AE, Brock DW. Deciding for Others: The Ethics of Surrogate Decision Making. New York, NY: Cambridge University Press; 1989.

2. In re Quinlan, 70 N.J. 10. 355 A. 2d 647 (1976).

3. Meisel A. The Right to Die. 2nd ed. New York, NY: John Wiley \& Sons, Inc; 1995.

4. Braun UK, Beyth RJ, Ford ME, McCullough LB. Voices of African American, Caucasian, and Hispanic surrogates on the burdens of end-of-life decision-making. J Gen Intern Med. 2008;23(3):267-274.

5. Faden RR, Beauchamp TL. A History and Theory of Informed Consent. New York, NY: Oxford University Press; 1986.

6. Byrne JP. The development of decision-making. J Adolesc Health. 2002;31(6 Suppl):208-215.

7. Locke EA, Latham GP. A Theory of Goal Setting and Task Performance. Englewood Cliffs, NJ: Prentice Hall; 1990.

8. Locke EA, Latham GP. Building a practically useful theory of goal setting and task motivation. A 35-year odyssey. Am Psychol. 2002;57(9):705-717.

9. Levinson W, Kao A, Kuby A, Thisted RA. Not all patients want to participate in decision making: a national study of public preferences. J Gen Intern Med. 2005;20(6):531-535.

10. Torke AM, Alexander GC, Lantos J, Siegler M. The physician-surrogate relationship. Arch Intern Med. 2007;167(11):1117-1121.

11. Berger JT, DeRenzo EG, Schwartz J. Surrogate decision making: reconciling ethical theory and clinical practice. Ann Intern Med. 2008;149(1):48-53.

12. Torke AM, Alexander GC, Lantos J. Substituted judgment: the limitations of autonomy in surrogate decision making. J Gen Intern Med. 2008;23(9):1514-1517. 